\title{
P01-11-26 Poster session
}

\section{The effect of sex on the pharmacokinetics/pharmacodynamics: A systematic review}

\section{Seonghae Yoon}

Seoul National University Bundang Hospital, Korea

Background

It is known that intrinsic factors such as age, sex, body weight, disease status, and organ functions, affect the pharmacokinetics (PK) and pharmacodynamics (PD) of many drugs. To select right drug and optimal dosage regimen of the drug considering these factors including genomics is key component of precision medicine. Even though sex is one of the variables that could lead to different drug efficacy or safety, clinical researches evaluating sex differences were not sufficient enough. In this study, we performed a systematic review to evaluate the effect of sex on the PK and PD of many drugs.

Methods

A comprehensive search of MEDLINE (PubMed), EMBASE was conducted. The articles published from 2014 to Sep 2017 was included in this study. We used 'sex, gender, pharmacokinetics' as search terms. We included gender as a search term, because many articles are using 'gender' instead of sex even though they meant the biological characteristics. Studies were included according to the following criteria: (1) prospective or retrospective studies on PK and/or PD of drugs in human, (2) studies included sex (or gender) as an independent variable, (3) studies with accessible full text We excluded studies according to the following criteria: (1) a case report, an editorial, or a review, (2) studies with no sufficient information on the sex differences.

Results

We reviewed titles and abstracts of 2070 articles were found that was published from 2014 to Sep 2017. Among those articles, 290 studies included the evaluation of sex differences in the PK and/or PD. The most common drug categories in ATC system were drugs targeting nervous system, antiinfectives, antineoplastic and immunomodulating agents, alimentary tract and metabolism, and cardiovascular system. More than half of the studies (about 57\%) revealed that there are differences in PK characteristics and/or PD between male and female.

Concolusions

We confirmed that more than half of the drugs searched in this study showed different pharmacokinetics characteristics between men and women. We'll need to do more researches to reveal the extent and mechanism of these difference between male and female and use those information on daily practice. 